\title{
Myocardial rescue with autologous mitochondrial transplantation in a porcine model of ischemia/reperfusion
}

\author{
Aditya K. Kaza, MD, ${ }^{\text {a }}$ Isaac Wamala, MD, ${ }^{a}$ Ingeborg Friehs, MD, ${ }^{a}$ Joseph D. Kuebler, MD, ${ }^{b}$ \\ Rahul H. Rathod, MD, ${ }^{\mathrm{b}}$ Ignacio Berra, MD, ${ }^{\mathrm{a}}$ Maria Ericsson, BS, ${ }^{\mathrm{c}}$ Rouan Yao, BS, ${ }^{\mathrm{d}}$ \\ Jerusha K. Thedsanamoorthy, BS, ${ }^{\mathrm{d}}$ David Zurakowski, PhD, ${ }^{\mathrm{d}}$ Sidney Levitsky, MD, \\ Pedro J. del Nido, MD, ${ }^{\mathrm{a}}$ Douglas B. Cowan, PhD, ${ }^{\mathrm{d}}$ and James D. McCully, PhD ${ }^{\mathrm{a}}$
}

\begin{abstract}
Objective: To demonstrate the clinical efficacy of autologous mitochondrial transplantation in preparation for translation to human application using an in vivo swine model.
\end{abstract}

Methods: A left mini-thoracotomy was performed on Yorkshire pigs. The pectoralis major was dissected, and skeletal muscle tissue was removed and used for the isolation of autologous mitochondria. The heart was subjected to regional ischemia (RI) by temporarily snaring the circumflex artery. After 24 minutes of $\mathrm{RI}$, hearts received $8 \times 0.1 \mathrm{~mL}$ injections of vehicle (vehicle-only group; $\mathrm{n}=6$ ) or vehicle containing mitochondria (mitochondria group; $n=6$ ) into the area at risk (AAR), and the snare was released. The thoracotomy was closed, and the pigs were allowed to recover for 4 weeks.

Results: Levels of creatine kinase-MB isoenzyme and cardiac troponin I were significantly increased $(P=.006)$ in the vehicle-only group compared with the mitochondria group. Immune, inflammatory, and cytokine activation markers showed no significant difference between groups. There was no significant between-group difference in the AAR $(P=.48)$, but infarct size was significantly greater in the vehicle group $(P=.004)$. Echocardiography showed no significant differences in global function. Histochemistry and transmission electron microscopy revealed damaged heart tissue in the vehicle group that was not apparent in the mitochondria group. T2-weighted magnetic resonance imaging and histology demonstrated that the injected mitochondria were present for 4 weeks.

Conclusions: Autologous mitochondrial transplantation provides a novel technique to significantly enhance myocardial cell viability following ischemia and reperfusion in the clinically relevant swine model. (J Thorac Cardiovasc Surg 2017;153:934-43)

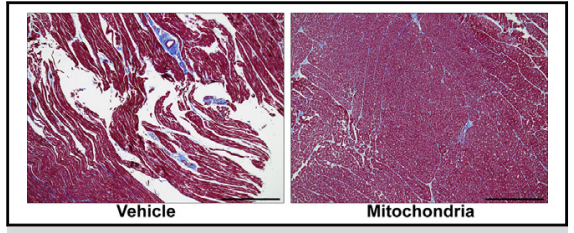

Autologous mitochondrial transplantation decreases infarct size and preserves structure.

\section{Central Message}

Autologous mitochondrial transplantation provides a novel technique to significantly enhance myocardial cell viability following ischemia and reperfusion.

\section{Perspective}

Autologous mitochondrial transplantation is an efficacious cardioprotective therapy as demonstrated in the clinically relevant swine model. The isolation of autologous mitochondria can be performed in $>30$ minutes, within the time frame associated with cardiac surgery. Direct injection of autologous mitochondria into the area at risk significantly decreases markers of myocardial injury and infarct size.

See Editorial Commentary page 944.

\footnotetext{
From the Departments of ${ }^{\mathrm{a}}$ Cardiac Surgery, ${ }^{\mathrm{b}}$ Cardiology, and ${ }^{\mathrm{d}}$ Anesthesiology, Perioperative and Pain Medicine, Boston Children's Hospital, 'Electron Microscopy Facility, Department of Cell Biology, and ${ }^{\mathrm{e}}$ Division of Cardiac Surgery, Beth Israel Deaconess Medical Center, Harvard Medical School, Boston, Mass.

This work was supported by Boston Scientific Corporation, a Richard A. and Susan F. Smith President's Innovation Award, Boston Children's Hospital, Michael B. Klein and family, and the Sidman Family Foundation.

Read at the 96th Annual Meeting of The American Association for Thoracic Surgery, May 14-18, 2016, Baltimore, Maryland.

Received for publication March 23, 2016; revisions received Oct 6, 2016; accepted for publication Oct 20, 2016; available ahead of print Dec 6, 2016

Address for reprints: James D. McCully, PhD, Department of Cardiac Surgery, Boston Children's Hospital, 300 Longwood Ave, Enders Building 407, Boston, MA 02115 (E-mail: james_mccully@hms.harvard.edu).

0022-5223/\$36.00

Copyright (c) 2016 by The American Association for Thoracic Surgery

http://dx.doi.org/10.1016/j.jtcvs.2016.10.077
}

In a series of previous studies, we have demonstrated that ischemia detrimentally alters mitochondrial structure, volume, calcium accumulation, complex activity, oxygen consumption, high-energy synthesis, mitochondrial-mediated intrinsic apoptosis, mitochondrial DNA integrity, and mitochondrial transcript and proteomics. ${ }^{1-5}$ All of these events

Scanning this QR code will take you to the article title page. To view the AATS 2016 Webcast, see the URL next to the video thumbnail. 


$$
\begin{aligned}
& \text { Abbreviations and Acronyms } \\
& \begin{aligned}
\text { AAR } & =\text { area at risk } \\
\text { CK-MB } & =\text { creatine kinase-MB isoenzyme } \\
\text { cTnI } & =\text { cardiac troponin I } \\
\text { IL } & =\text { interleukin } \\
\text { LV } & =\text { left ventricular } \\
\text { MRI } & =\text { magnetic resonance imaging } \\
\text { RI } & =\text { regional ischemia }
\end{aligned}
\end{aligned}
$$

occur during ischemia and extend into reperfusion to severely compromise postischemic cellular viability. ${ }^{1-3,6}$

We have postulated that the augmentation or the addition of healthy mitochondria would allow for enhanced postischemic cellular viability and overcome the effects of stunning. In preliminary experiments, we used isolated perfused rabbit hearts and in situ blood-perfused rabbit hearts with 2 hours and 4 weeks of recovery. ${ }^{7,8}$ These investigations demonstrated that the transplantation of autologous mitochondria significantly decreases infarct size and significantly increases postischemic functional recovery. ${ }^{7,8}$ The transplanted mitochondria act both extracellularly and intercellularly to enhance oxygen consumption, increase high-energy phosphate synthesis, and induce cytokine mediators and proteomic pathways important for preserving myocardial energetics, cell viability, and enhanced postinfarct cardiac function.

The transplanted mitochondria are initially located in close proximity to myocardial cells and by 1 to 4 hours are integrated into the myocardial cells by actin-dependent endocytosis both as clusters and as individual mitochondria, where they rescue mitochondrial function and repair damaged mitochondrial DNA. ${ }^{8,9}$ The transplanted mitochondria are functional and viable for at least 4 weeks. ${ }^{8,9}$ The injected mitochondria do not elicit any immune or autoimmune response and are not proarrhythmic. ${ }^{8}$

To allow for clinical relevance, here we report the results of our present study using a clinically relevant in situ large animal (swine) model.
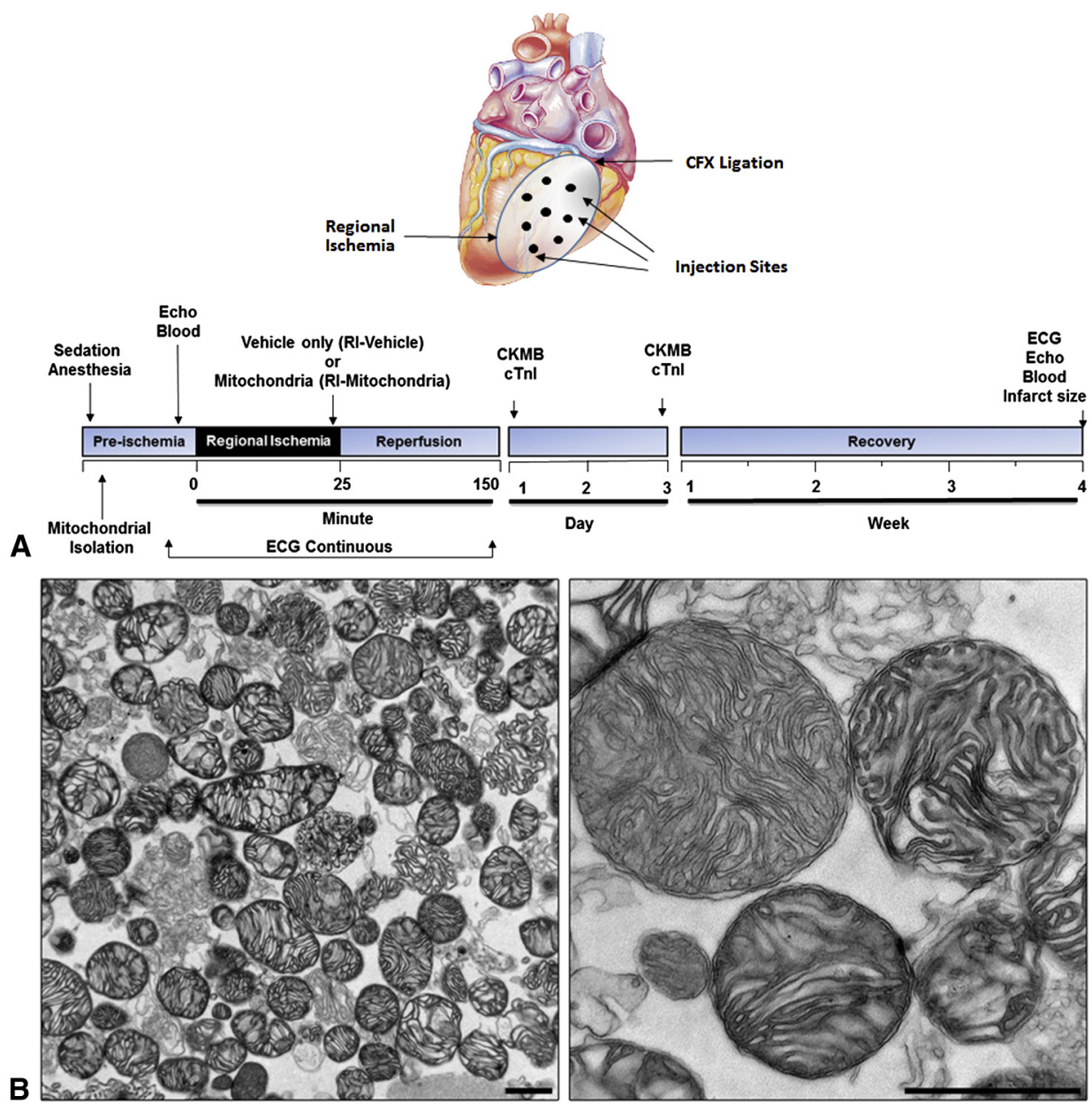

FIGURE 1. A, Experimental protocol. Injection sites in the area at risk are indicated. B, Representative electron micrographs of isolated pig skeletal muscle mitochondria. The isolated mitochondria were free from cellular contamination and were electron dense and had preserved morphology and shape. (Scale bars: $1 \mu \mathrm{m}$.) RI, Regional ischemia; ECG, echocardiography. 


\section{MATERIALS AND METHODS Animal Care and Biosafety}

Yorkshire pigs (females, $35-45 \mathrm{~kg} ; \mathrm{n}=20$ ) were used for all experimental studies. Four pigs in the vehicle-only group died during recovery after arterial ligation due to intractable ventricular fibrillation and were excluded from our analysis. Twelve pigs (vehicle only, $n=6$; mitochondria, $\mathrm{n}=6$ ) survived for 4 weeks. Four additional pigs underwent magnetic resonance imaging (MRI). This investigation was conducted in accordance with the National Institutes of Health's guidelines on animal care and use and was approved by the Boston Children's Hospital's Animal Care and Use Committee (Protocol 15-01-2722). All animals received humane care in compliance with the Guide for the Care and Use of Laboratory Animals (http://www.nap.edu/catalog.php?record_ $\mathrm{id}=12910)$.

\section{Surgical Protocol}

The pig was sedated with telazol $(2.2-4.4 \mathrm{mg} / \mathrm{kg})$ and xylazine $(1-2 \mathrm{mg} / \mathrm{kg})$ through intramuscular injection. An endotracheal tube was placed, and general anesthesia was induced with isoflurane (induction, $3 \%$; maintenance, $0.5 \%-2 \%$ ). Core temperature was maintained at $>36^{\circ} \mathrm{C}$ using a heating pad. Continuous invasive blood pressure through a femoral artery line and continuous pulse oximetry were monitored as well. Lidocaine $(2 \mathrm{mg} / \mathrm{kg})$ via continuous intravenous infusion was administered from the start of the thoracotomy, and heparin $(100 \mathrm{U} / \mathrm{kg})$ was given as a bolus before the snaring of the circumflex artery.

A small left thoracotomy was performed through the fourth intercostal space. The pectoralis major muscle was isolated, and 2 small muscle tissue samples (approximately $0.03 \mathrm{~g}$ ) were removed using a \#6 biopsy punch (Miltex, York, Pa) and stored for mitochondrial isolation under sterile conditions. The pericardium was opened, and the circumflex artery was located. A Prolene 3-0 suture (Ethicon, Somerville, NJ) was passed around the artery with a taper needle, and both ends of the tie were threaded through a small vinyl tube to form a snare. The artery was temporarily occluded by pulling the snare and fixed by clamping the tube with a mosquito clamp. RI was induced for 25 minutes and confirmed visually by ST-segment changes on the electrocardiogram. ${ }^{8}$

\section{Mitochondrial Isolation}

Mitochondria were isolated from the pectoralis major muscle. ${ }^{10,11}$ The isolated mitochondria were suspended in $1 \mathrm{~mL}$ of respiration buffer containing $250 \mathrm{mmol} / \mathrm{L}$ sucrose, $2 \mathrm{mmol} / \mathrm{L} \mathrm{KH}_{2} \mathrm{PO}_{4}, 10 \mathrm{mmol} / \mathrm{L} \mathrm{MgCl}_{2}$, $20 \mathrm{mmol} / \mathrm{L} \mathrm{K}^{+}$-HEPES [4-(2-hydroxyethyl)-1-piperazineethanesulfonic acid] buffer $\mathrm{pH} 7.2,0.5 \mathrm{mmol} / \mathrm{L} \mathrm{K}^{+}$-EGTA (ethylene glycol tetra-acetic acid) $\mathrm{pH} 8.0,5 \mathrm{mmol} / \mathrm{L}$ glutamate, $5 \mathrm{mmol} / \mathrm{L}$ malate, $8 \mathrm{mmol} / \mathrm{L}$ succinate, and $1 \mathrm{mmol} / \mathrm{L}$ adenosine diphosphate. ${ }^{8}$ Mitochondrial viability was assessed as described previously. ${ }^{8}$

\section{Experimental Groups}

After 24 minutes of RI, hearts were randomized to receive either eight 0.1 -mL injections of sterile respiration buffer (vehicle) or eight 0.1 - $\mathrm{mL}$ injections of sterile respiration buffer containing mitochondria $(9.9 \times$ $10^{7} \pm 1.4 \times 10^{7} / \mathrm{mL} ; 1.3 \times 10^{7}$ mitochondria per injection site) into the area at risk (AAR) (Figure 1). Each injection was delivered subendocardially into predesignated sites within the AAR using a sterile $1-\mathrm{mL}$ insulin syringe with a 28 -gauge needle. ${ }^{7,8}$

At 25 minutes of RI, the snare was released, and the pigs were then allowed to recover for 4 weeks. The Prolene suture-snare was trimmed and loosely tied and left in place to identify the AAR and infarct size. ${ }^{8}$ The pericardium was closed, pleural air was evacuated, and the thoracotomy incision was closed. ${ }^{8}$ Thirty minutes before the end of the procedure, a fentanyl patch ( $4 \mathrm{mg} / \mathrm{kg}$, transdermal for 72 hours) was applied for pain prophylaxis.
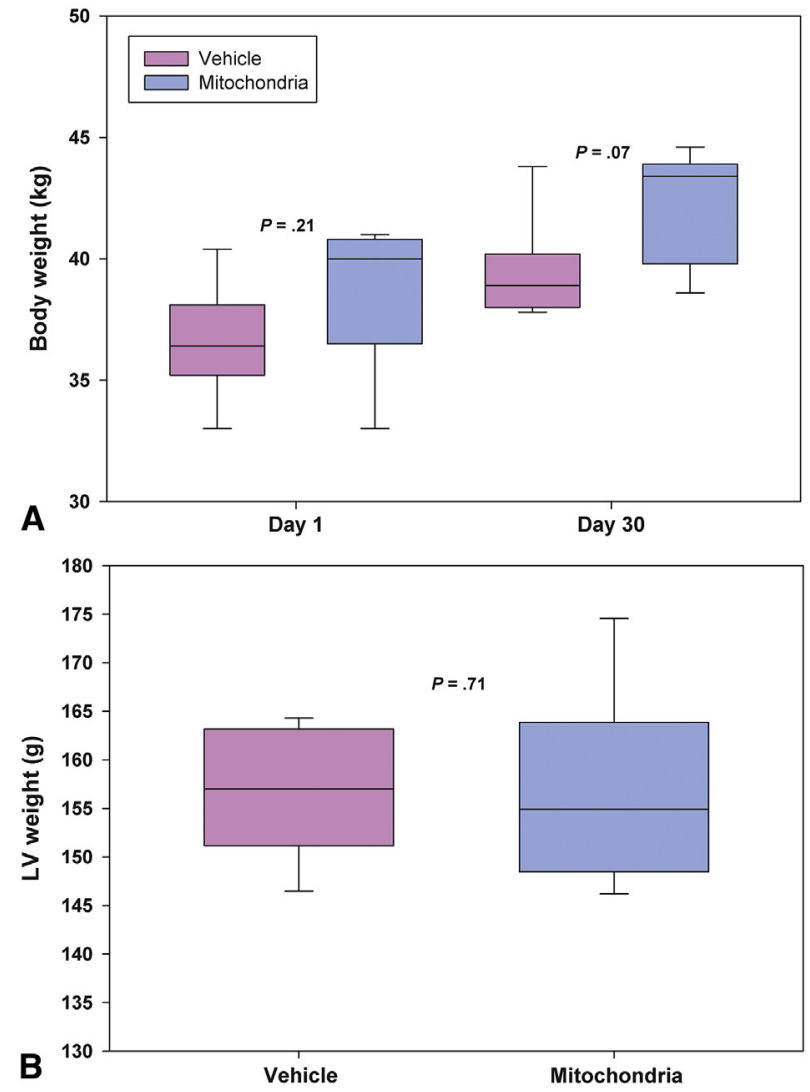

FIGURE 2. Body weight (A) and heart weight (B). There was no significant difference in body weight between groups either at the time of initial or terminal surgery. The mean change in body weight over 4 weeks $(\delta)$ is shown in (A). There was no significant difference in left ventricular weight at the time of initial or terminal surgery (B). $L V$, Left ventricular.

\section{Echocardiography}

Echocardiography was performed using a Philips iE33 machine with a 5-MHz transducer (Philips Healthcare, Amsterdam, The Netherlands). The pigs were sedated and placed in the left lateral position. Left ventricular (LV) measurements were obtained on parasternal short-axis and long-axis images. Circumferential strain analysis was performed offline with TomTec 2D Cardiac Performance Analysis version 1.0 (TomTec Imaging Systems, Munich, Germany).

\section{Blood Samples}

Blood samples were collected in serum-separating tubes (BD Vacutainer SST; BD, Franklin Lakes, NJ) and stored at $-80^{\circ} \mathrm{C}$ until use in biomarker analysis. ${ }^{8}$

\section{Euthanasia}

At 4 weeks of recovery, the pigs were sedated and anesthetized as described above. Then a sternotomy was performed, the heart was removed for biochemical and histological analysis, and the animal was euthanized by exsanguination.

\section{Measurement of AAR and Infarct Size}

The AAR and infarct size were measured as described previously. ${ }^{12}$

\section{Histology and Electron Microscopy}

Tissue samples from the AAR were dissected out and used for histology and electron microscopy analyses. ${ }^{7,8}$ 


\section{Markers of Myocardial Injury}

Creatine kinase (CK-MB) and cardiac troponin I (cTnI) concentrations were estimated using a swine-specific enzyme-linked immunosorbent assay (Biocheck, Foster City, Calif; Life Diagnostics, West Chester, Pa).

\section{Multiplex Assay}

Serum samples from baseline and at 4 weeks of recovery were used for cytokine analysis using the Porcine Cytokine/Chemokine Kit and the TGF- $\beta 1$ Array (EMD Millipore, St Charles, Mo). Multiplex assays were performed at Eve Technologies (Calgary, Alberta, Canada) using the Bio-Plex 200 system (Bio-Rad Laboratories, Hercules, Calif). All samples and standards were run in duplicate.

\section{MRI}

In a separate study, 4 pigs were used for MRI studies. Each pig was sedated and anesthetized, a mini-thoracotomy was performed, the heart was exposed, and RI was induced as described above. In brief, human cardiac fibroblasts were maintained in Fibroblast Medium-2 (ScienCell Reasearch Laboratories, Carlsbad, Calif) according to the supplier's directions and used for mitochondrial isolation. The mitochondria were labeled at $0^{\circ} \mathrm{C}$ with $10 \mathrm{mg}$ of $N$-succinimidyl ester-functionalized 30-nm magnetic iron (II, III) oxide particles (Sigma-Aldrich, St Louis, Mo) in mitochondrial respiration buffer for 10 minutes, and then washed 3 times in the same buffer containing $1 \mathrm{mg} / \mathrm{mL}$ fraction $\mathrm{V}$ bovine serum albumin (Sigma-Aldrich).

After 24 minutes of RI, each heart received eight 0.1 -mL injections of sterile respiration buffer containing human cardiac fibroblast mitochondria labeled with iron $\left(1.0 \times 10^{8} / \mathrm{mL}\right)$. The chests were closed, and the pigs were allowed to survive for 4 weeks. At 4 weeks, the hearts were removed and perfusion-fixed under constant pressure using $4 \%$ paraformaldehyde in phosphate-buffered saline ( $\mathrm{pH}$ 7.4) and imaged on a BioSpec 70/30 USR 7-T MRI System (Bruker, Billerica, Mass). High-resolution 3-dimensional T2-weighted images were acquired using fast low-angle shot MRI.

\section{Histology and Microscopy}

Ventricles from fixed pig hearts used for T2*-weighted MRI were embedded in paraffin and sectioned for histological examination. Rehydrated tissue sections ( $5 \mu \mathrm{m}$ thick) were subjected to antigen retrieval in $1 \mathrm{mmol} / \mathrm{L}$ ethylenediaminetetraacetic before incubation with the anti-mitochondria mouse monoclonal antibody [MTC02] (Abcam; ab3298) and anti-desmin rabbit monoclonal antibody (Abcam; ab32362). Primary antibodies were detected with species-appropriate secondary antibodies conjugated to Alexa Fluor 488 or 568 dyes (Thermo Fisher Scientific) and cross-stained with 4',6-diamidino-2-phenylindole (DAPI) (Thermo-Fisher). Mounted sections were visualized on a FSX100 inverted fluorescence microscope (Olympus). Brightfield microscopy of sections stained using the Iron Stain Kit (Sigma-Aldrich, St. Louis MO) was performed with the same microscope.

\section{Statistical Analysis}

Statistical analyses were performed using SAS version 9.2 (SAS Institute, Cary, NC). To reduce the risk of false positive conclusions (type I errors) due to multiple testing, we applied the Bonferroni method using the multcomp package in $\mathrm{R}$ for vehicle versus mitochondria comparisons regarding CK-MB, CTnI, and infarct size ${ }^{13,14}$ We did not adjust the $P$ values for multiplicity regarding cytokine activation, because we did not expect group differences in these markers based on our hypotheses.

\section{RESULTS}

\section{Body and Heart Weights}

There was no significant difference $(P=.21)$ in animal body weight between the vehicle only $(\mathrm{n}=6)$ and mitochondria groups $(\mathrm{n}=6)$ after 4 weeks of recovery (Figure 2, A). The median of the $\delta$ for body weight was $1.9 \mathrm{~kg}$ (IQR, 0.9-5.6 kg) for the vehicle group and $3.6 \mathrm{~kg}$ (IQR, 2.6-5.1 kg) for the mitochondria group $(P=.62)$.

Mean LV weight following 4 weeks of recovery was $156.7 \pm 7.4 \mathrm{~g}$ in the vehicle group and $158.8 \pm 9.7 \mathrm{~g}$ in the mitochondria, a statistically nonsignificant difference $(P=.71)$ (Figure 2, $B)$

\section{Immune Response and Cytokine Activation}

Immune and inflammatory response and cytokine activation, as measured by a multiplex assay (Milliplex

TABLE 1. Cytokine activation levels at 30 days after surgery

\begin{tabular}{|c|c|c|c|}
\hline Variable & Vehicle $(\mathrm{n}=6), \mathrm{pg} / \mathrm{mL}$, median (range) & Mitochondria $(\mathrm{n}=6), \mathrm{pg} / \mathrm{mL}$, median $($ range) & $P$ value* \\
\hline GM-CSF & $1(1-26)$ & $1(1-1)$ & .66 \\
\hline IFN- $\gamma$ & $1(1-1)$ & $1(1-1)$ & 1 \\
\hline IL-1a & $24(4-178)$ & $17(1-234)$ & .66 \\
\hline IL-1B & $104(49-3195)$ & $752(73-7394)$ & .73 \\
\hline IL-1ra & $715(403-3584)$ & $362(282-1671)$ & .18 \\
\hline IL-2 & $30(1-1342)$ & $113(1-2697)$ & 1 \\
\hline IL-4 & $492(184-12,392)$ & $1955(10-4021)$ & .69 \\
\hline IL-6 & $419(380-459)$ & $69(1-1214)$ & .38 \\
\hline IL-8 & $1(1-1)$ & $1(1-9)$ & .67 \\
\hline IL-10 & $110(29-1063)$ & $161(48-3859)$ & .84 \\
\hline IL-12 & $1325(316-1679)$ & $1339(968-3574)$ & .54 \\
\hline IL-16 & $881(436-4482)$ & $2018(580-11,120)$ & .66 \\
\hline TNF- $\alpha$ & $35(12-73)$ & $27(16-81)$ & 1 \\
\hline TGF- $\beta 1$ & $24,028(15,787-31,932)$ & $16,924(11,486-27,075)$ & .49 \\
\hline TGF- $\beta 2$ & $22,110(16,136-40,291)$ & $22,212(14,177-48,551)$ & 1 \\
\hline TGF- $\beta 3$ & $244(240-364)$ & $418(350-574)$ & .06 \\
\hline
\end{tabular}

$G M$-CSF, Granulocyte-macrophage colony-stimulating factor; $I F N$, interferon; $I L$, interleukin; $T N F$, tumor necrosis factor; $T G F$, transforming growth factor. *Groups compared using the Mann-Whitney $U$ test. 

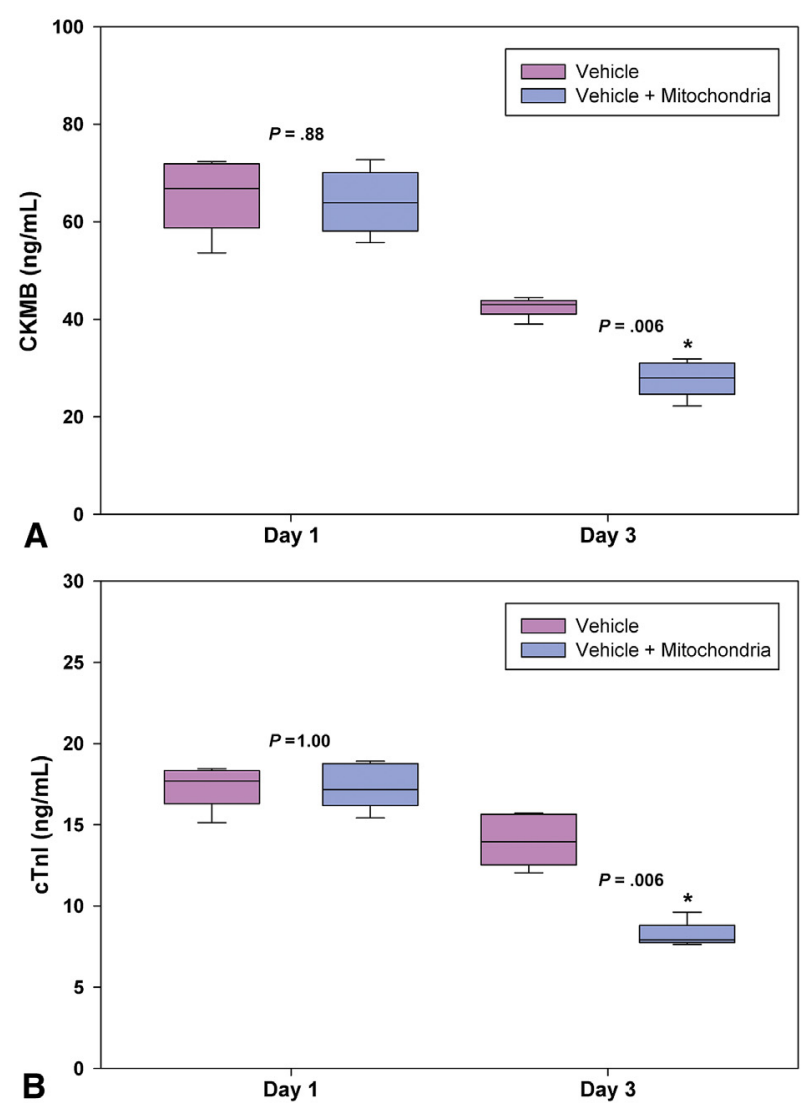

FIGURE 3. Creatine kinase-MB isoenzyme (CK-MB) (A) and cardiac troponin I (cTnI) (B) at day 1 and day 3 after surgery in the vehicle-only and mitochondria groups. Both CK-MB and cTnI were significantly decreased $(P<.001)$ in mitochondria hearts compared with vehicle hearts at day 3 . There was no significant difference between the 2 groups at day 1 . Results are shown for $\mathrm{n}=6$ in each group. Significant differences compared with vehicle are shown. *Significant differences compared with vehicle at $P=.006$.

Porcine Cytokine/Chemokine Kit, Millipore, St Charles, MO), showed no significant difference between groups after 4 weeks of recovery (Table 1 ).

\section{Markers of Myocardial Injury}

There were no significant between-group differences in CK-MB (median: vehicle, 66.9 [IQR, 58.8-71.9]; mitochondria, 63.9 [IQR, 58.1-70.1]; $P=.88$ ) (Figure 3, A), or cTnI (median: vehicle, 17.7 [IQR, 16.3-18.3]; mitochondria, 17.2 [IQR, 16.2-18.8]; $P=1.000$ ) immediately after surgery (Figure 3, $B$ ).

At 3 days after surgery, CK-MB was significantly lower in the mitochondria group compared with the vehicle group (median: vehicle, 43.1 [IQR, 41.1-43.9]; mitochondria, 28.0 [IQR, 24.6-31.0]; $P=.006$ ) (Figure 3, $A$ ). Similarly, cTNi at 3 days after surgery was significantly lower in the mitochondria group (median: vehicle, 14.0 [IQR, 12.5-15.7]; mitochondria, 7.9 [IQR, 7.7-8.8]; $P=.006$ ) (Figure 3, B). These results indicate significantly greater myocardial damage in the vehicle-only group compared with the mitochondria group.

\section{Area at Risk and Infarct Size}

There was no significant difference in the median AAR (\% LV mass) between the 2 groups (vehicle: $28.5 \%$ [IQR, 25.6\%-31.8\%]; mitochondria: $33.2 \%$ [IQR, $25.0 \%-36.4 \%$ ]; $P=.48$ ) (Figure $4, A)$.

Median infarct size ( $\%$ AAR $)$ was significantly greater in the vehicle group compared with the mitochondria group $(13.0 \%$ [IQR, $10.9 \%-19.0 \%]$ vs $5.2 \%$ [IQR, $4.2 \%-7.0 \%$ ]; $P=.004$ ) (Figure $4, B$ ).

Histochemistry and electron microscopy. Histochemical microscopy analysis of samples from the regional ischemic area showed significantly increased longitudinal and transverse interfibrillar separation in vehicle hearts compared with mitochondria hearts (Figure 4,C). There was no alteration in collagen content observable between groups. Electron microscopy analysis showed mitochondrial damage and contraction bands in vehicle hearts that were not present in mitochondria hearts (Figure 4,D).

Electrocardiography. Electrocardiograms showed no difference between groups prior to ischemia; however, with the onset of regional ischemia, ST segment elevations $(>1 \mathrm{~mm})$ consistent with myocardial infarction were observed in all hearts (Figure 5, A). Electrocardiographic recordings at 120 minutes after injection of vehicle or mitochondria and at 4 weeks recovery showed no ventricular tachycardia, bradycardia, fibrillation, conduction system defects, mitral regurgitation or repolarization heterogeneity (Figure 5, A).

\section{Echocardiography}

Echocardiography showed no significant differences in the end-diastolic volume or parameters of global systolic function (ejection fraction, shortening fraction, and global circumferential strain) (Figure 5, B).

\section{Myocardial Uptake of Mitochondria}

To demonstrate mitochondrial uptake, pig hearts were injected with mitochondria isolated from human cardiac fibroblast cell cultures and then labeled with iron. Figure 6, $A$ shows MRI images of mitochondria uptake in pig hearts at 4 weeks after injection. Mitochondria were verified using antibodies specific to human mitochondria (Figure 6, B). Our results show that mitochondria are present for at least 4 weeks after injection in the pig heart.

\section{DISCUSSION}

Here we have demonstrated the efficacy of autologous mitochondrial transplantation in a clinically relevant porcine model. We used a mini-thoracotomy and obtained fresh nonischemic tissue from the pectoralis major; however, any nonischemic tissue source may be used. The mass of 

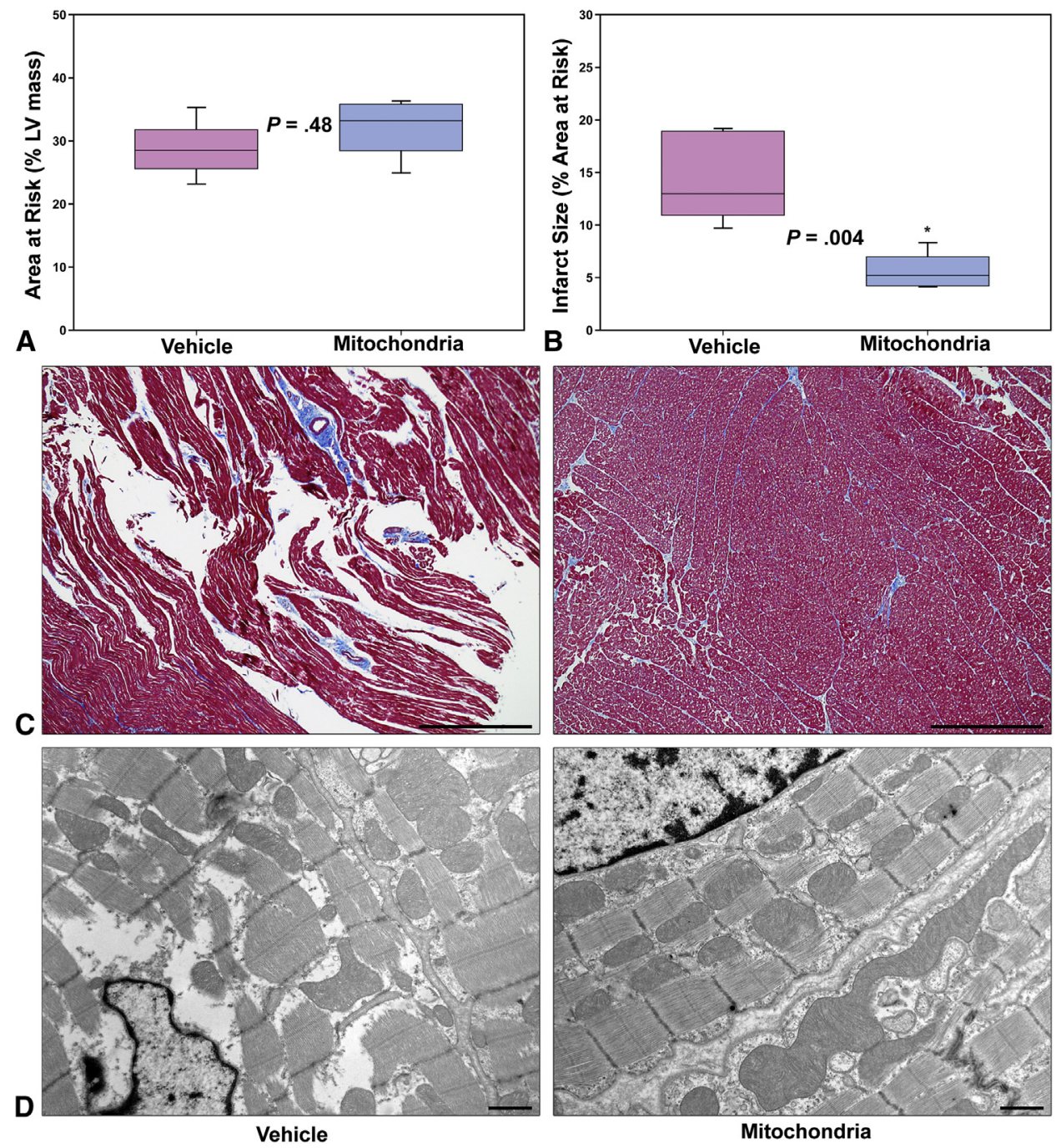

FIGURE 4. A and B, Area at risk (AAR) (A) and infarct size (\%AAR) (B) for vehicle and mitochondria. Results are shown as the mean \pm standard deviation for $\mathrm{n}=6$ in each group. Significant differences compared with vehicle are shown. C and D, Histochemical and electron microscopy analysis of myocardial tissue at 4 weeks of recovery. Representative Masson's trichrome-stained (C) and transmission electron (D) micrographs from vehicle and mitochondria hearts are shown. Longitudinal and transverse interfibrillar separation was observed in vehicle hearts but not in mitochondria hearts (C), but there was no difference in collagen between the 2 groups (C). Electron microscopy analysis (D) shows mitochondrial damage and contraction bands in vehicle hearts that are not present in mitochondria hearts. (Scale bars: $500 \mu \mathrm{m}$.) $L V$, Left ventricular.

tissue required is small and is obtained using either scissors or a \#6 biopsy punch. Dissection using electrocauterization is not recommended (personal communication).

To provide for clinical use, we have optimized the isolation of mitochondria. The isolation of mitochondria is rapid and can be performed in less than 30 minutes, a reasonable time frame within most cardiac surgical operations. The tissue required is small and can be obtained using a \#6 dermatology punch. It is important to note that the source of mitochondria is unimportant in allowing for cardioprotection. In previous studies, we demonstrated that total, interfibrillar, and subsarcolemmal mitochondria isolated from the heart, liver, or skeletal muscle provide similar cardioprotection. ${ }^{7}$ A step-by-step visual demonstration of the isolation procedure with quality assurance parameters is available elsewhere. ${ }^{10,11}$

We tested doses of $2 \times 10^{5}, 2 \times 10^{6}, 2 \times 10^{7}$, and $2 \times 10^{8}$ mitochondria per $1 \mathrm{~g}$ of tissue (wet weight). These preliminary studies demonstrated no significant difference in the decrease in infarct size despite the differences in mitochondria number injected. In all of our studies, we suspended the mitochondria in a final volume of $1 \mathrm{~mL}$ and injected at 8 to 10 sites within the AAR. Mitochondrial concentrations $>2 \times 10^{8}$ were not entirely suspended in $1 \mathrm{~mL}$ of buffer, and thus these concentrations were not used. ${ }^{15}$ The number of mitochondria used in these studies 


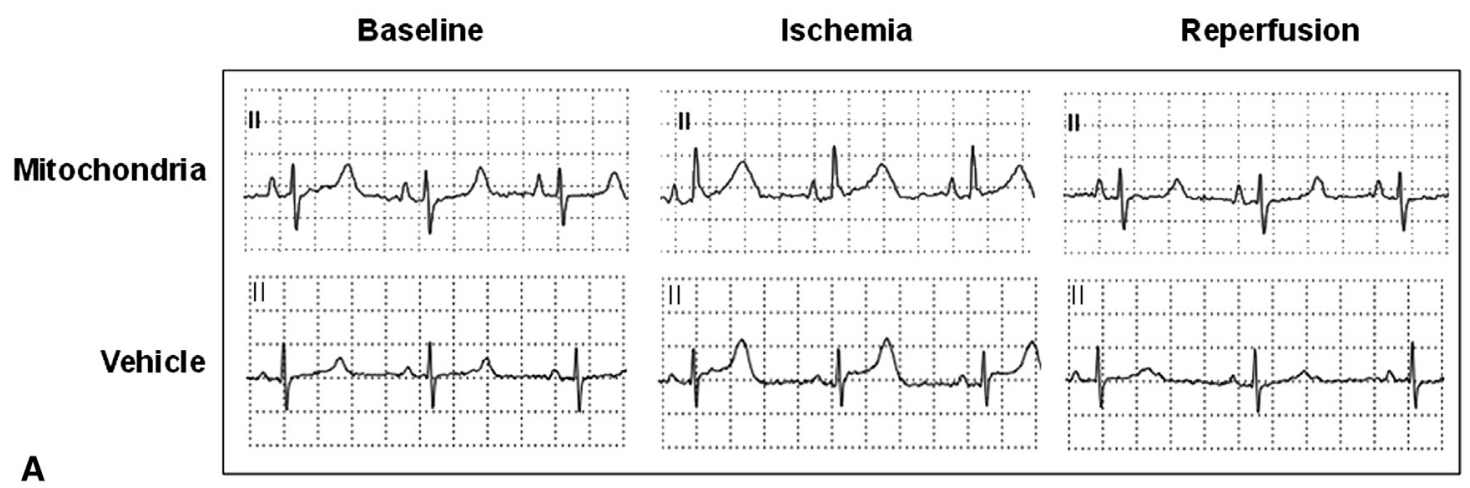

A

A

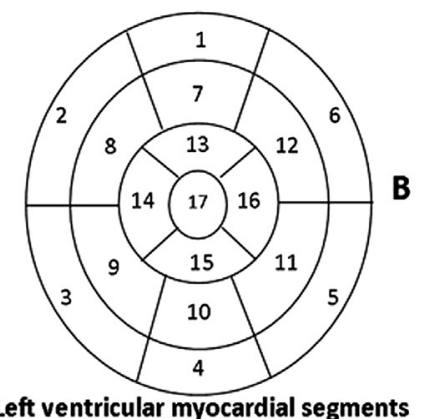

\begin{tabular}{|c|c|c|c|}
\hline & Vehicle (\%) & Mitochondria (\%) & P Value \\
\hline GCS & $-22.0 \pm 1.4$ & $-22.1 \pm 3.0$ & 0.29 \\
\hline Anterior (7) & $-16.9 \pm 4.6$ & $-20.2 \pm 5.4$ & 0.54 \\
Anteroseptal (8) & $-24.1 \pm 3.0$ & $-24.1 \pm 5.4$ & 0.35 \\
\hline Inferoseptal (9) & $-29.4 \pm 9.7$ & $-21.3 \pm 5.0$ & 0.28 \\
\hline Inferior (10) & $-22.3 \pm 12.6$ & $-21.5 \pm 8.3$ & 0.67 \\
\hline Inferolateral (11) & $-16.2 \pm 6.9$ & $-24.3 \pm 6.1$ & 0.31 \\
\hline Anterolateral (12) & $-17.8 \pm 4.1$ & $-21.3 \pm 4.9$ & 0.57 \\
\hline
\end{tabular}

FIGURE 5. A, Electrophysiological data. Representative electrocardiograms at baseline (before surgery), ischemia (following 10 minutes of regional ischemia), and reperfusion (following 120 minutes of reperfusion). B, Echocardiography data. A, Recommended 17-segment model for the left ventricle. ${ }^{14}$ $\mathrm{B}$, Global and regional circumferential strain analysis from vehicle and mitochondria hearts. Strain was measured in the midventricular short-axis plane (segments 7-12). More negative strain values indicate a more favorable strain. Values are shown as mean \pm standard deviation.

was $9.9 \times 10^{7} \pm 1.4 \times 10^{7} / \mathrm{mL}$, delivered as eight $0.1-\mathrm{mL}$ injections within the area at risk $\left(1.3 \times 10^{7}\right.$ mitochondria per injection site). Injections were made using a sterile 1$\mathrm{mL}$ insulin syringe with a 28 -gauge needle. ${ }^{7,8}$

The injected mitochondria did not induce electrical disturbances. No ventricular tachycardia, bradycardia, fibrillation, conduction system defects, or repolarization heterogeneity was observed. ${ }^{8}$ Our results also show that the injected mitochondria do not elicit any immune or inflammatory responses. There was no up-regulation of cytokines associated with the immune or inflammatory response seen in patients with acute heart transplantation rejection (interleukin [IL]-1, IL-4, IL-6, IL-12, IL-18, interferon- $\gamma$-inducible protein 10 , and macrophage inflammatory proteins $1 \alpha$ and $1 \beta$ ), and there was no overproduction of transforming growth factor $\beta 1, \beta 2$, or $\beta 3 .^{16,17}$

Of importance, our findings demonstrate that the intramyocardial injection of autologous mitochondria into the AAR of the ischemia/reperfusion-injured heart significantly decreases CK-MB and cTnI concentrations and significantly decreases infarct size. The effects of mitochondrial injection on infarct size persist for at least 4 weeks after injection.

To demonstrate that the transplanted mitochondria are present in the myocardium for 4 weeks after injection, we used a separate group of pigs for mitochondria experiments as described above, but using xenogeneic human mitochondrial isolated from human cardiac fibroblast primary cell cultures. This use of human mitochondria in a pig model allowed for the differentiation between native pig mitochondria and transplanted human mitochondria based on immune reactivity to a monoclonal anti-human mitochondria antibody in postmortem histological tissue analysis. Our results show that injected mitochondria are taken up by myocardial cells and are present in the myocardium for at least 4 weeks after injection. In a separate study, we reported that the mean fraction of mitochondria taken up by the myocardium was $76.18 \% \pm 11.85 \%{ }^{18}$

The mechanisms through which the mitochondrial transplantation provides for cardioprotection involve energy synthesis, cell rescue, replacement of damaged mitochondrial DNA, up-regulation of protein pathways for the mitochondrion and the generation of precursor metabolites for energy and cellular respiration, and up-regulation of cytokines shown to play key roles in angiogenesis, arteriogenesis, immunomodulation, progenitor cell migration, prevention of apoptosis, and enhanced cell salvage and postischemic functional recovery. ${ }^{7-9}$ These mechanisms act at the time of injection and persist in the myocardium for at least 4 weeks (the duration of our present investigation). 

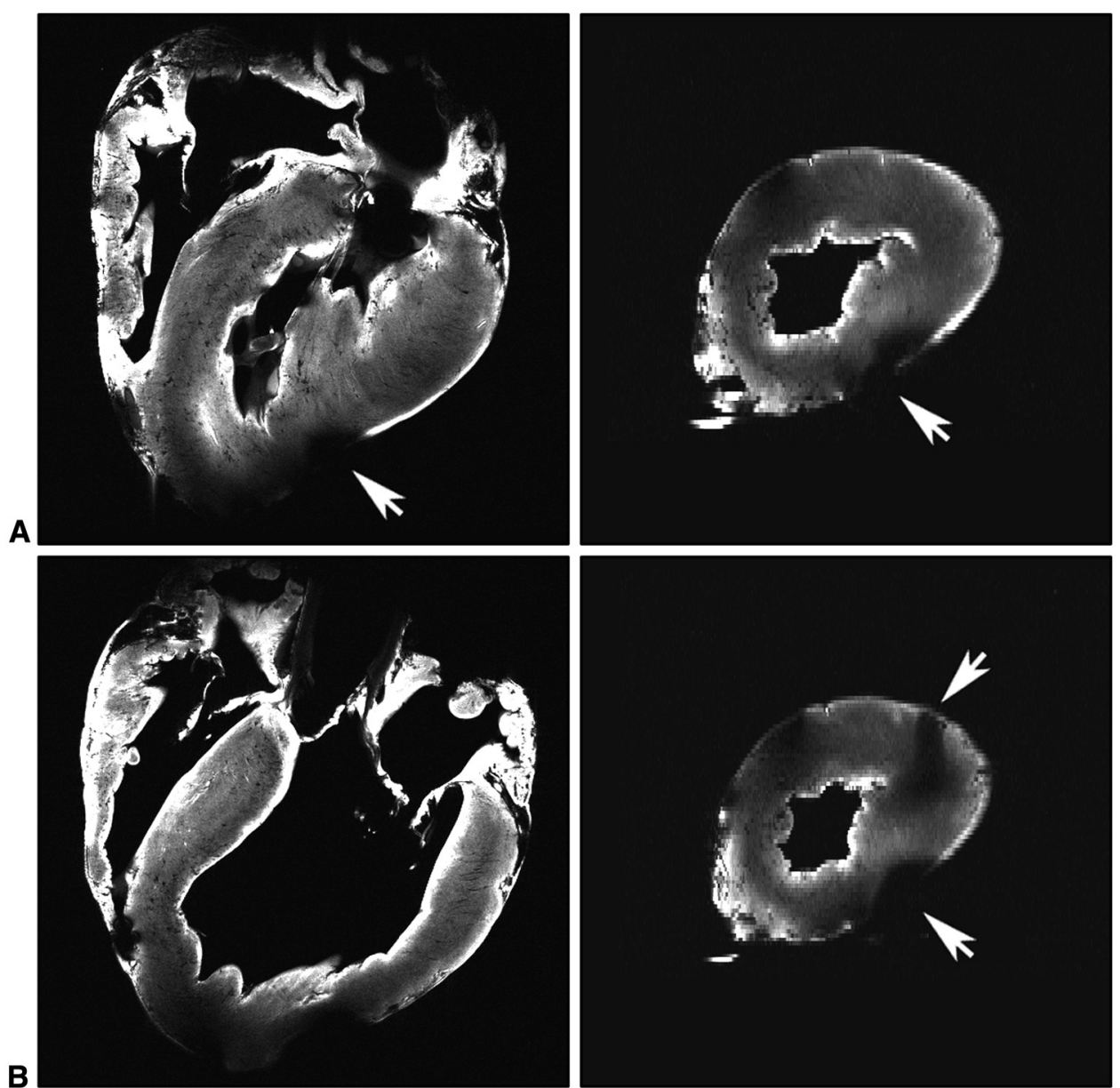

B
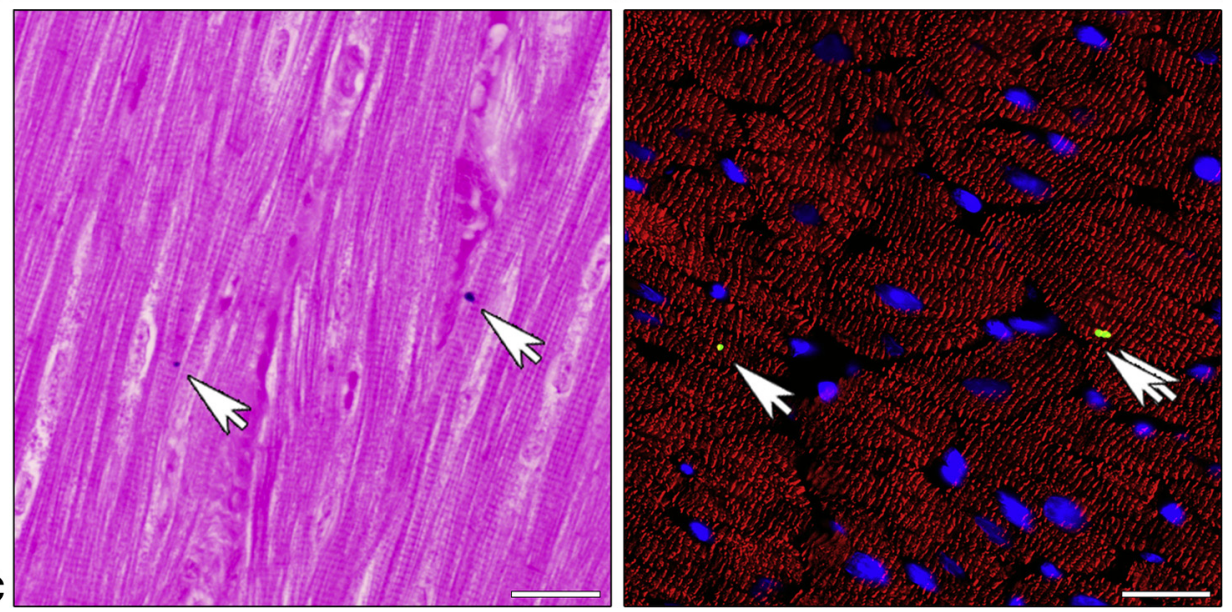

FIGURE 6. Mitochondrial uptake at 4 weeks after injection. A and B, T2*-weighted magnetic resonance imaging of mitochondrial uptake in pig heart at 4 weeks after injection. Iron oxide-labeled mitochondria appear as dark regions in the heart (arrows). Coronal (left) and transverse slices (right) are depicted from 2 different regions of the same heart. C, Histological sections from the dark regions were stained for iron (left) and human mitochondria (right). Our results show that mitochondria are present for at least 4 weeks after injection in the pig heart.

Mitochondrial transplantation is a simple process that can be used following restoration of coronary flow or following reparative cardiac surgery. In this study, we injected the mitochondria directly into the myocardium; however, we recently demonstrated that vascular delivery can be used as well. ${ }^{18}$ 


\section{Limitations}

This study used a model of acute myocardial infarction in which treatment is immediate and without added complications. In this laboratory setting, the rescue of myocardial cells following ischemia and associated stunning is optimal, and thus our results may be overly optimistic in a clinical setting in which there may be a delay in the response to treatment.

In this study, we used the circumflex artery rather than the left anterior descending (LAD) artery for ligation. We did so to allow for relevant evaluation of the efficacy of autologous mitochondrial injection for cardioprotection. Initial studies using the LAD demonstrated that the animals receiving vehicle only were severely injured and did not survive. To avoid death as an endpoint, we reasoned that a less severe but still clinically relevant injury would provide more relevant and useful data.

We were unable to demonstrate any significant betweengroup differences in global myocardial function using serial parasternal echocardiography. ${ }^{19}$ The use of parasternal echocardiography proved difficult, given the small and irregular echocardiology window in the pig owing to its large and extended sternum and large muscle mass. These anatomic features hinder consistent measurements and comparison of results between groups. ${ }^{20}$ In previous studies, we used epicardial echocardiography to demonstrate differences between groups. In the present study, we did not use epicardial echocardiography, to avoid possible induction of fibrillation in the more sensitive pig heart. These differences most likely contributed to our inability to detect significant differences in end-diastolic volume or parameters of global systolic function despite the significant differences in infarct size between hearts receiving vehicle only and those receiving autologous mitochondria.

\section{CONCLUSIONS}

As demonstrated in our clinically relevant swine model, autologous mitochondrial transplantation is an efficacious cardioprotective therapy. The isolation of mitochondria is rapid and can be performed within the clinically relevant time frame associated with cardiac surgery. No proarrhythmic, immune, or inflammatory reactions were observed following mitochondrial transplantation. The transplanted mitochondria remained in the myocardium for at least 4 weeks after injection.

\footnotetext{
Webcast

You can watch a Webcast of this AATS meeting presentation by going to: http://webcast.aats.org/2016/ Video/Tuesday/05-17-16_Ballroom_IV_0730_McCully800.mp4.
}

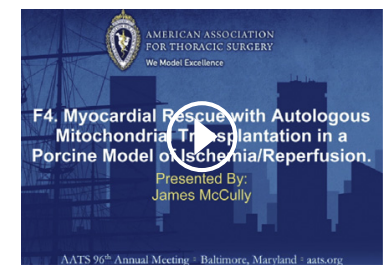

\section{Conflict of Interest Statement}

Dr McCully received experimental support from Boston Scientific Corporation to pay for these studies. Drs McCully, Cowan, Levitsky, and have patents pending for the isolation and usage of mitochondria. All other authors have nothing to disclose with regard to commercial support. The authors attest that they had complete freedom to explore the data, analyze the results independent from any sponsor, and sole authority to make the final decision to submit the material for publication.

We thank Arthur Nedder, DVM, Elizabeth Pollack, BA, Naomi Crilley, BS, and Courtney White, BS, LATG, of Animal Resources at Children's Hospital, Boston Children's Hospital, for their assistance with the surgeries reported in this article.

\section{References}

1. Tsukube T, McCully JD, Metz KR, Cook CU, Levitsky S. Amelioration of ischemic calcium overload correlates with high-energy phosphates in the senescent myocardium. Am J Physiol Heart Circ Physiol. 1997;273: H418-25.

2. McCully JD, Wakiyama H, Hsieh YJ, Jones M, Levitsky S. Differential contribution of necrosis and apoptosis in myocardial ischemia/reperfusion injury. Am J Physiol Heart Circ Physiol. 2004;286:H1923-35.

3. Rousou AJ, Ericsson M, Federman M, Levitsky S, McCully JD. Opening of mitochondrial KATP channels enhances cardioprotection through the modulation of mitochondrial matrix volume, calcium accumulation and respiration. Am J Physiol Heart Circ Physiol. 2004;287:H1967-76.

4. McCully JD, Rousou AJ, Parker RA, Levitsky S. Age- and gender-related differences in mitochondrial oxygen consumption and calcium with cardioplegia and diazoxide. Ann Thorac Surg. 2007;83:1102-9.

5. Levitsky S, Laurikka J, Stewart RD, Campos CT, Lahey SJ, McCully JD. Mitochondrial DNA deletions in coronary artery bypass grafting patients. Eur J Cardiothorac Surg. 2003;24:777-84.

6. Lesnefsky EJ, Chen Q, Slabe TJ, Stoll MS, Minkler PE, Hassan MO, et al. Ischemia, rather than reperfusion, inhibits respiration through cytochrome oxidase in the isolated, perfused rabbit heart: role of cardiolipin. Am J Physiol Heart Circ Physiol. 2004;287:H258-67.

7. McCully JD, Cowan DB, Pacak CA, Toumpoulis IK, Dayalan H, Levitsky S. Injection of isolated mitochondria during early reperfusion for cardioprotection. Am J Physiol Heart Circ Physiol. 2009;296:H94-105.

8. Masuzawa A, Black KM, Pacak CA, Ericsson M, Barnett RJ, Drumm C, et al. Transplantation of autologously-derived mitochondria protects the heart from ischemia-reperfusion injury. Am J Physiol Heart Circ Physiol. 2013;304: H966-82.

9. Pacak CA, Preble JM, Kondo H, Seibel P, Levitsky S, Del Nido PJ, et al. Actindependent mitochondrial internalization in cardiomyocytes: evidence for rescue of mitochondrial function. Biol Open. 2015;4:622-6.

10. Preble JM, Pacak CA, Kondo H, McKay AA, Cowan DB, McCully JD. Rapid isolation and purification of mitochondria for transplantation by tissue dissociation and differential filtration. J Vis Exp. 2014;e51682.

11. Preble JM, Kondo H, Levitsky S, McCully JD. Quality control parameters for mitochondria transplant in cardiac tissue. JSM Biochem Mol Biol. 2014;2:1008.

12. Wakiyama H, Cowan DB, Toyoda Y, Federman M, Levitsky S, McCully JD. Selective opening of mitochondrial ATP-sensitive potassium channels during 
surgically induced myocardial ischemia decreases necrosis and apoptosis. Eur J Cardiothorac Surg. 2002;21:424-33.

13. $\mathrm{R}$ Development Core Team. $R$ : a language and environment for statistical computing. Vienna, Austria: $\mathrm{R}$ Foundation for Statistical Computing; 2013.

14. Bretz F, Hothorn T, Westfall P. Multiple comparisons using R. Boca Raton (FL): Chapman and Hall/CRC Press; 2011: 38-81.

15. McCully JD, Levitsky S, Del Nido PJ, Cowan DB. Mitochondrial transplantation for therapeutic use. Clin Transl Med. 2016;5:16.

16. Lim H, Zhu YZ. Role of transforming growth factor-beta in the progression of heart failure. Cell Mol Life Sci. 2006;63:2584-96.

17. Rosenkranz S. TGF-beta1 and angiotensin networking in cardiac remodeling. Cardiovasc Res. 2004;63:423-32.

18. Cowan DB, Yao R, Akurathi V, Snay ER, Thedsanamoorthy JK, Zurakowski D, et al. Intracoronary delivery of mitochondria to the ischemic heart for cardioprotection. PLoS One. 2016;11:e0160889.

19. Cerqueira MD, Weissman NJ, Dilsizian V, Jacobs AK, Kaul S, Laskey WK, et al. Standardized myocardial segmentation and nomenclature for tomographic imaging of the heart. A statement for healthcare professionals from the Cardiac Imaging Committee of the Council on Clinical Cardiology of the American Heart Association. Circulation. 2002;105:539-42.

20. Lee MY, Lee SH, Lee SG, Park SH, Lee CY, Kim KH, et al. Comparative analysis of heart functions in micropigs and conventional pigs using echocardiography and radiography. $J$ Vet Sci. 2007;8:7-14.

Key Words: infarct, ischemia, mitochondria, reperfusion

\section{Discussion}

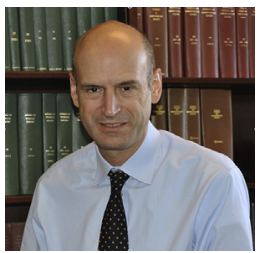

Dr Juan A. Crestanello (Columbus, Ohio). I would like to thank the Society for the opportunity to discuss this paper. I also would like to congratulate Dr McCully and colleagues for a well-designed set of experiments and for an excellent presentation.

In this experiment, the authors expanded their research on mitochondrial transplantation to a large animal model of ischemic heart disease. In a pig model of myocardial ischemia-reperfusion achieved by 25 minutes of regional ischemia, autologous skeletal muscle mitochondria were injected in the experimental group versus vehicle in the control. Hearts were recovered for 4 weeks. Myocardial infarction, size, and enzyme release were decreased in the mitochondrial group, although it resulted in no difference in left ventricular ejection fraction detected by echo. Transplanted mitochondria were presented up to 4 weeks after the injections.

The overall hypothesis of this set of experiments is that the mitochondria controls the fate of the cells in the ischemic myocardium, and the transplanted healthy mitochondria into the myocardium will decrease myocardial ischemia-reperfusion injury.

I have the following questions. The injections were performed at the end of the ischemic period. Was there any difference if the injections were performed earlier?

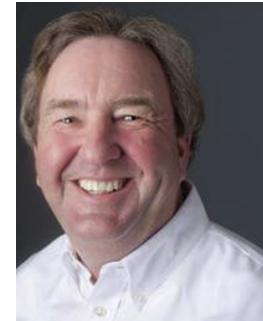

Dr McCully. We didn't want to inject the mitochondria earlier, because then they would have been damaged by the ischemic phase. Mitochondria can be injected during the reperfusion phase; they seem to work during that phase also.

Dr Crestanello. So were the mitochondria really incorporated into the cells? Do you have any idea where they were located?

Dr McCully. Up to 1 hour following injection, the mitochondria act extracellularly. They increase high-energy stores and alter the proteomic profile to energy metabolism. At 10 minutes to 1 hour, the mitochondria are internalized by the cells, where they increase the ATP within the cells and repair the damaged mitochondrial DNA.

Dr Crestanello. So were the mitochondria alive at 4 weeks, were you able to determine that on the autologous mitochondria, because in your paper you used human mitochondria?

Dr McCully. That is a good question. It is very difficult to tell whether autologous mitochondria that you injected are functioning, because you can't tell the autologous mitochondria from the mitochondria that are resident within the cell. We have used human mitochondria for in vitro studies and have done in vitro assays where the mitochondria function for 23 days, 53 half-lives in HeLa cells. So I would have to say yes, there is no reason for them not to.

Dr Crestanello. Finally, can you speculate on why this happened, what is the mechanism? If we follow the path of stem cell transplantation initially in the research, it was thought that it was a mechanistic effect of the cells improving contraction. Following an analogy, is this related to improved myocardial bioenergetics by the mitochondria, or is it because of the effects on apoptosis?

Dr McCully. It is all of those. The first effects are to increase intercellular ATP. Within 10 minutes we get a complete change in the proteomic profile. There is a shift over the mitochondria; mitochondrial synthesis, mitochondrial biogenesis. Within one hour the injected mitochondria are taken up by the cells; they repair the damaged mitochondrial DNA and they increase the cellular ATP content.

Dr Crestanello. Thank you and congratulations, again, for outstanding work.

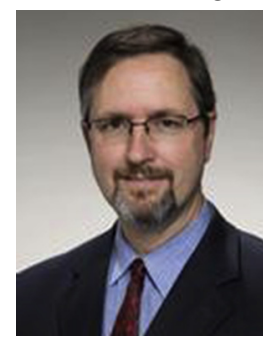

Dr Frank W. Sellke (Providence, $R I$ ). In your purification process, are you getting rid of all microvesicles or exosomes, because similar results have been reported using those entities?

Dr McCully. Frank, we have documented that the preparation of isolated mitochondria is pure by enzymatic activity, antibody identification, oxygen consumption, and transmission electron microscopy. There are no contaminants in the preparation. 